\title{
Incidence and Predictors of Nephrotoxicity Associated with Intravenous Colistin in Overweight and Obese Patients
}

\author{
Timothy P. Gauthier, ${ }^{\text {a }}$ William R. Wolowich, ${ }^{\text {a }}$ Arathi Reddy, ${ }^{b}$ Ennie Cano, ${ }^{c}$ Lilian Abbo, ${ }^{\text {d }}$ and Laura B. Smith ${ }^{c}$ \\ Department of Pharmacy Practice, Nova Southeastern University College of Pharmacy, Fort Lauderdale, Florida, USA; Department of Pharmacy, West Kendall Baptist

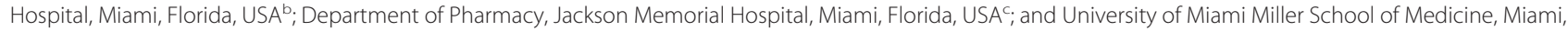 \\ Florida, USA
}

Intravenous colistin is used to treat resistant Gram-negative infections and is associated with nephrotoxicity. In overweight and obese adults, a paucity of data exists regarding the incidence and predictors of such toxicity. A retrospective nested case-control study was performed over 35 months for patients receiving intravenous colistin for $\geq 72 \mathrm{~h}$ with a body mass index (BMI) of $\geq 25$ $\mathrm{kg} / \mathrm{m}^{2}$. The objective was to investigate the incidence and predictors of nephrotoxicity. Severity of acute kidney injury was defined by RIFLE (risk, injury, failure, loss, and end-stage kidney disease) criteria. Dosing and mortality were secondarily investigated. Forty-two patients met the inclusion criteria, and $20(48 \%)$ developed nephrotoxicity. Patients with toxicity were in the risk (15\%), injury (5\%), and failure (80\%) categories based on RIFLE criteria. A logistic regression model identified four predictors of colistin-associated nephrotoxicity: a BMI of $\geq 31.5 \mathrm{~kg} / \mathrm{m}^{2}$ (odds ratio [OR], 3.1; 95\% confidence interval [CI], 1.15 to 8.35), diabetes (OR, 2.11; 95\% CI, 0.84 to 5.29), the length of hospitalization in days prior to receipt of colistin (OR, 1.04; 95\% CI, 0.99 to 1.08$)$, and age (OR, 1.08; $95 \%$ CI, 1.00 to 1.17). Among all of the patients, dosing based on the actual body weight and excessive dosing due to the use of the actual body weight were frequent at $64 \%$ and $92 \%$, respectively. The 30 -day all-cause in-hospital mortality rate was $40 \%$ in the toxicity group and $14 \%$ in the nontoxicity group $(P=0.14)$. Patients receiving intravenous colistin should be monitored for nephrotoxicity, especially when the BMI exceeds $31.5 \mathrm{~kg} / \mathrm{m}^{2}$. Prospective, randomized, controlled trials are warranted to further examine nephrotoxicity incidence and predictors and appropriate dosing strategies in this population.

ntravenous colistimethate sodium (CMS, colistin) is a polymyxin-type antimicrobial currently used to treat multidrug-resistant (MDR) Gram-negative infections caused by bacteria such as Acinetobacter baumannii, Pseudomonas aeruginosa, and Klebsiella pneumoniae (19). Although it was first used in the 1950s, CMS fell into disuse primarily due to significant associated nephrotoxicity (NTX), with incidence reports now ranging from $0 \%$ to $53.5 \%$ (8). Its unique history has been described in multiple reviews (8, $18,21,24)$, and there is a notable lack of definitive recommendations regarding the most efficacious and least toxic way to administer CMS, including in overweight and obese patients (for whom the ideal body weight [IBW] is recommended by the package insert $[13,28])$. As the obesity epidemic continues within the United States, at a rate of $>33 \%$ among adults (10), the questions of what the risk factors for NTX are and how clinicians should administer CMS to these patients remain critical.

Limited data are available regarding NTX in overweight or obese patients receiving intravenous CMS. Following a thorough review of the literature, the only previously published evaluation of more than one overweight or obese individual receiving intravenous CMS included 10 obese patients (defined as those with an actual body weight [ABW] that is $>140 \%$ of the IBW) (4). This analysis found that excessive CMS dosing was frequent and that use of the ABW to calculate doses was associated with a higher rate of NTX. Other published literature including nonoverweight or nonobese populations has shown multiple potential risk factors associated with CMS use to include male sex, age, hypoalbuminemia, hyperbilirubinemia, long durations of therapy, higher cumulative CMS doses, receipt of concomitant nephrotoxins, and concomitant vancomycin, rifampin, or calcineurin inhibitors (12, 15-17, 23). Furthermore, NTX has been shown to independently predict fewer cures of infection and to increase the mortality rate (26).

Given the questions at hand, the objectives of this study were to investigate the incidence of and predictors of the development of NTX in overweight and obese patients receiving intravenous CMS therapy. Mortality and dosing were secondarily evaluated.

\section{MATERIALS AND METHODS}

A retrospective nested case-control study of adults (age, $\geq 18$ years) receiving intravenous CMS was performed at a university affiliated hospital between 1 January 2008 and 30 November 2010. This large tertiary-care center provides care to a dynamic patient population that includes medical, surgical, level 1 trauma, transplant, cancer, and critically ill patients. A pharmacy-generated report was used to identify patients who had received intravenous CMS during the study period. Patients were excluded if they had a body mass index (BMI) of $<25 \mathrm{~kg} / \mathrm{m}^{2}$, had undergone kidney transplantation, received CMS for $<72 \mathrm{~h}$, were receiving renal replacement therapy, had a baseline serum creatinine $\left(S_{C R}\right)$ of $>2 \mathrm{mg} / \mathrm{dl}$, or did not have at least two consecutive stable $\mathrm{S}_{\mathrm{CR}}$ measurements (an increase of no greater than 20\%) within $72 \mathrm{~h}$ of CMS initiation. Clinical data were obtained from electronic and paper medical records. Only one treatment (the first) per patient was considered in the analysis. The CMS used in this study was manufactured by X-Gen Pharmaceuticals, Inc., supplied as 150 mg CMS base activity per vial (28). CMS measurements are given as base

Received 5 January 2012 Returned for modification 9 January 2012 Accepted 8 February 2012

Published ahead of print 27 February 2012

Address correspondence to Timothy Gauthier, Timothy.Gauthier@nsu.nova.edu. Copyright $\odot$ 2012, American Society for Microbiology. All Rights Reserved. doi:10.1128/AAC.00028-12 
TABLE 1 Suggestions for modification of dosage schedules for adults with impaired renal function in package insert supplied with colistimethate for injection $^{a}$

\begin{tabular}{|c|c|c|c|c|}
\hline \multirow[b]{2}{*}{ Parameter } & \multicolumn{4}{|c|}{ Degree of impairment } \\
\hline & Normal & Mild & Moderate & Considerabl \\
\hline \multicolumn{5}{|l|}{ Renal function } \\
\hline $\begin{array}{l}\text { Plasma creatinine } \\
(\mathrm{mg} / 100 \mathrm{ml})\end{array}$ & $0.7-1.2$ & $1.3-1.5$ & $1.6-2.5$ & $2.6-4.0$ \\
\hline $\begin{array}{l}\text { Urea clearance } \\
\qquad \% \text { of normal })\end{array}$ & $80-100$ & $40-70$ & $25-40$ & $10-25$ \\
\hline \multicolumn{5}{|l|}{ Dosage } \\
\hline $\begin{array}{l}\text { Unit dose of colistimethate } \\
\text { for injection (mg) }\end{array}$ & $100-150$ & $75-115$ & $66-150$ & $100-150$ \\
\hline Frequency (times/day) & 4 to 2 & 2 & 2 or 1 & Every $36 \mathrm{~h}$ \\
\hline Total daily dose (mg) & 300 & $150-230$ & $133-150$ & 100 \\
\hline $\begin{array}{l}\text { Approximate daily dose } \\
(\mathrm{mg} / \mathrm{kg})\end{array}$ & 5 & $2.5-3.8$ & 2.5 & 1.5 \\
\hline
\end{tabular}

${ }^{a}$ Modified from references 13 and 28 with permission. In obese individuals, the dosage should be based on the IBW.

activity for the entirety of this report. Intravenous CMS dosing at this institution is not protocol driven; however, usual frequency and renal adjustments are reflected by recommendations from the package insert (Table 1). This study was approved by the local ethics committees, and written informed consent was not required.

NTX was defined as at least two consecutive $S_{C R}$ measurements with an increase of $0.5 \mathrm{mg} / \mathrm{dl}$ from the baseline at least $24 \mathrm{~h}$ apart after 2 or more days of CMS therapy. RIFLE (risk, injury, failure, loss, and end-stage kidney disease) criteria $(1,14)$ were used to define severity of acute kidney injury (Fig. 1). Classification of dosing regimens was based upon recommendations from the package insert, which includes modification for renal dysfunction. A daily dose was considered excessive, normal, or low normal if it was greater than, within, or below $\pm 0.4 \mathrm{mg} / \mathrm{kg} /$ day, respectively, of the recommended dosing range using the IBW $(4,6)$. The World Health Organization's international classifications, which are based on the BMI, were used to define overweight or obese patients as having a BMI of 25 to $29.9 \mathrm{~kg} / \mathrm{m}^{2}$ or $\geq 30 \mathrm{~kg} / \mathrm{m}^{2}$, respectively (27). Data collected on each patient included demographics, comorbid conditions (diabetes, peripheral vascular disease, congestive heart failure, transplant history), acute physiology and chronic health evaluation II (APACHE-II) score at CMS initiation, duration of CMS therapy, daily dose of CMS, $\mathrm{S}_{\mathrm{CR}}$ measurements on therapy and $72 \mathrm{~h}$ prior to therapy, serum albumin, total bilirubin, organism(s) causing infection, site of infection, 14- and 30-day all-cause in-hospital mortality, and length of hospitalization. Hypoalbuminemia was defined as a serum albumin level of $<2.0 \mathrm{~g} / \mathrm{dl}$, and hyperbilirubinemia was defined as a total bilirubin level of $>5 \mathrm{mg} / \mathrm{dl}$ (17). Evaluation of concomitant medications included vasopressors and nephrotoxic agents (intravenous radio contrast, angiotensin-converting enzyme inhibitors, angiotensin receptor blockers, aminoglycosides, rifampin, cyclosporine, nonsteroidal anti-inflammatory drugs sulfamethoxazole-trimethoprim, diuretics, tacrolimus, vancomycin, or antilymphocyte antibodies).

Data were screened for normality using a panel of seven tests. When data were normally distributed, descriptors of central tendency (mean), variance (standard deviation), and hypothesis testing were analyzed using parametric tests. For data that were not normally distributed, descriptors of central tendency (median), variance (interquartile range), and hypothesis testing were analyzed using nonparametric tests. Chi-square analysis was used to screen demographic and clinical factors known to contribute to NTX. Factors with a $P$ value of $<0.3$ were subsequently included in a forward-stepping with replacement multivariate logistic regression algorithm. All statistical procedures were carried out using NCSS v. 7.1.2.

\section{RESULTS}

Three hundred seventy unique patients were identified as receiving intravenous CMS during the study time period, 138 of whom were determined to be overweight or obese. When the exclusion criteria were applied to the overweight patients, 4 were excluded due to a history of kidney transplantation, 11 were excluded due to renal replacement therapy on day 1 of CMS therapy, 22 had a baseline $\mathrm{S}_{\mathrm{CR}}$ of $>2 \mathrm{mg} / \mathrm{dl}$, and 59 received CMS for $<72 \mathrm{~h}$. This resulted in 42 patients who met the inclusion criteria and were incorporated in the analysis. The median BMI was $31.9 \mathrm{~kg} / \mathrm{m}^{2}$, with a range of 26.4 to $87.7 \mathrm{~kg} / \mathrm{m}^{2}$. The characteristics of the cohort are listed in Table 2.

Twenty patients (48\%) experienced NTX during intravenous CMS therapy using the previously described definition. When NTX was defined as a decrease in baseline creatinine clearance (using the Salazar-Corcoran equation [25]) of $\geq 50 \%$ or $\geq 30 \%$, the NTX incidence was $28.6 \%$ or $47.6 \%$, respectively. Patients experiencing NTX were in the risk (15\%), injury (5\%), and failure $(80 \%)$ categories based on RIFLE criteria. There was a median of 5 days to the occurrence of NTX (range, 3 to 18 days), and the time to NTX is represented by Fig. 2. Of the 20 patients with NTX, 5 had undergone dialysis.

In a bivariate analysis, age $(P=0.04), \mathrm{ABW}(P=0.02), \mathrm{BMI}$ $(P=0.015)$, length of hospitalization in days before receipt of CMS $(P=0.008)$, and diabetes $(P=0.02)$ were found to predict the risk of the development of NTX while on CMS therapy (Table 2). Amphotericin B, rifampin, antilymphocyte antibodies, nonsteroidal anti-inflammatory drugs, and calcineurin inhibitors were not found to be given in this cohort. No concomitant nephrotoxins were found to have statistical significance.

In a multivariate analysis, the best logistic model (Table 3) included a BMI of $\geq 31.5 \mathrm{~kg} / \mathrm{m}^{2}$ (OR [odds ratio] 3.1, $P=0.025$ ), age (OR 1.08, $P=0.045$ ), diabetes (OR 2.11, $P=0.112$ ), and length of hospitalization prior to receipt of CMS (OR 1.04, $P=$ 0.078 ) as independent predictors of NTX. Age and body mass measurements, including BMI, were nonlinearly related to NTX (Fig. 3).

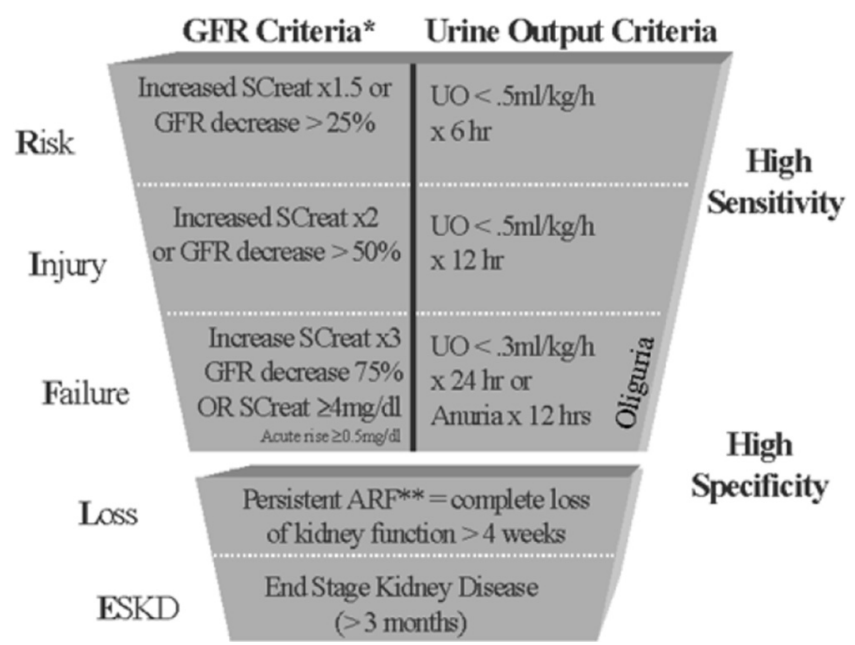

FIG 1 The RIFLE criteria for acute kidney injury(reproduced with permission from reference 1; originally published by BioMed Central). Abbreviations: SCreat, $\mathrm{S}_{\mathrm{CR}}$ concentration; GFR, glomerular filtration rate; UO, urine output; $\mathrm{ARF}$, acute renal failure. ${ }^{*}$, All $\mathrm{S}_{\mathrm{CR}}$ references are based on changes from the baseline values. 
TABLE 2 Bivariate analysis of risk factors for colistin-associated NTX

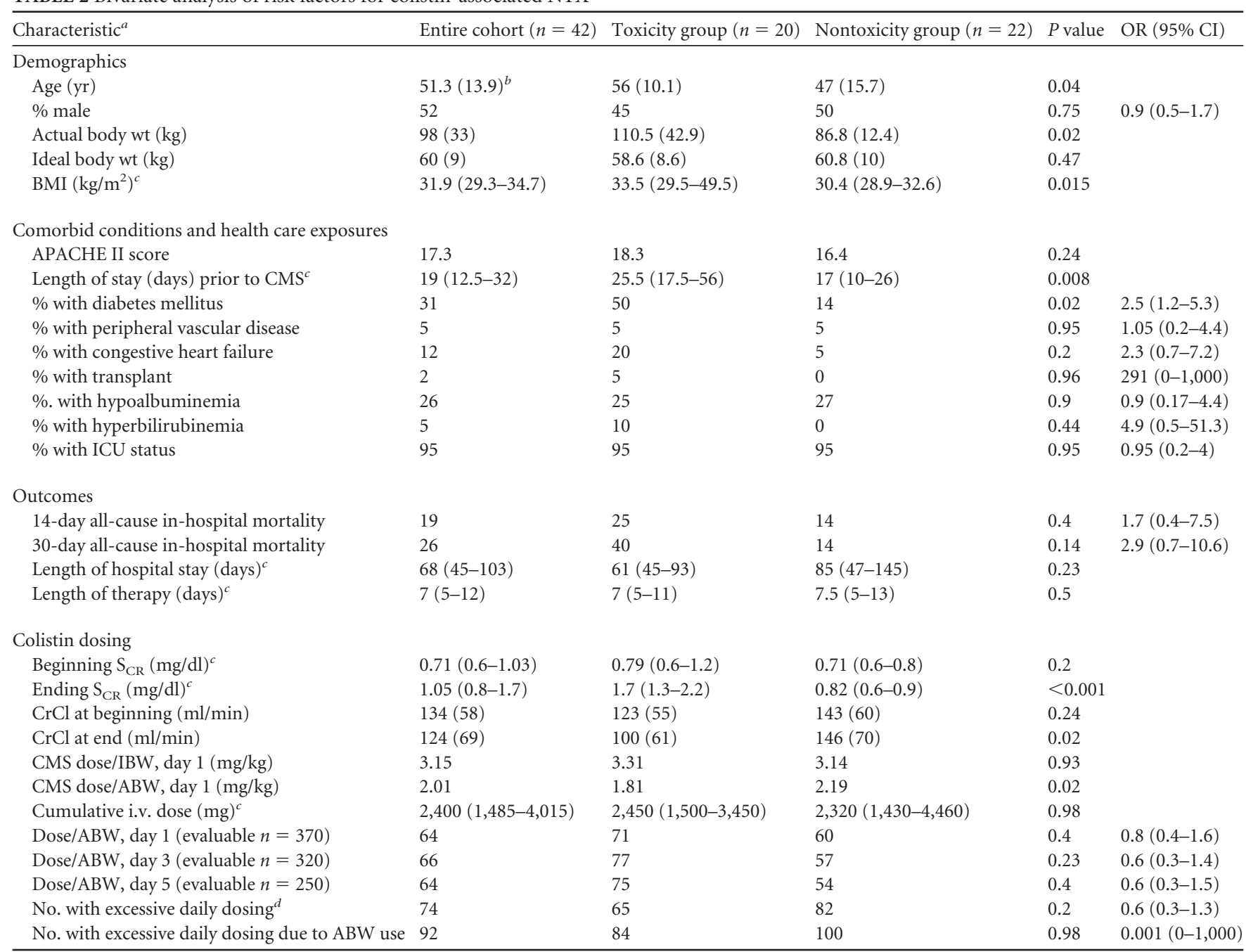

${ }^{a}$ Abbreviations: ICU, intensive care unit; $\mathrm{CrCl}$, creatinine clearance as calculated by the Salazar-Corcoran equation (25).

${ }^{b}$ All data represent mean percentages (standard deviations), unless otherwise indicated.

${ }^{c}$ Median (25th to 75th percentile interquartile range).

${ }^{d}$ No patients were found to have a low-normal daily dose.

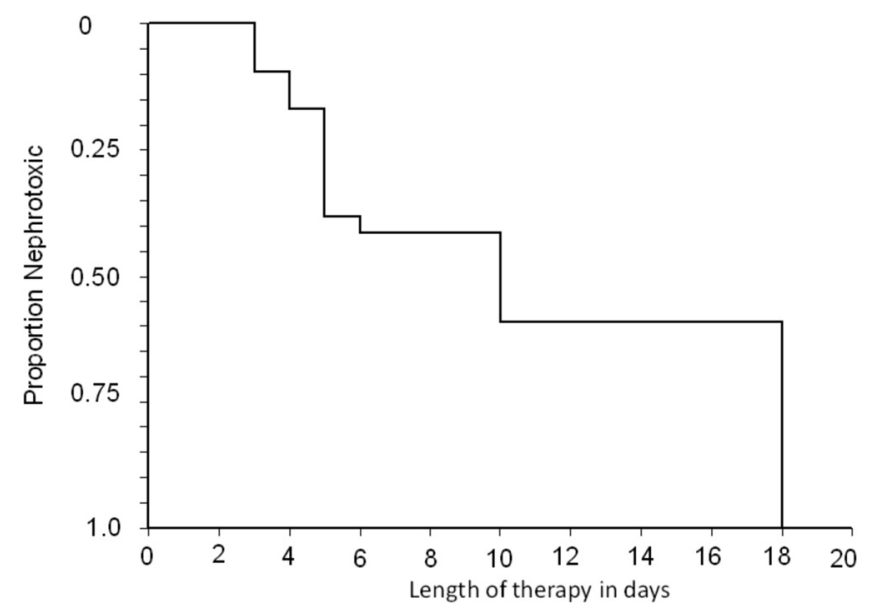

FIG 2 Days of intravenous CMS therapy to development of NTX.
The in-hospital 14-day all-cause mortality rate in the NTXpositive group was $25 \%$ versus $14 \%$ in the NTX-negative group $(P=0.4)$ (Table 2$)$. The 30 -day mortality rate was $40 \%$ in the NTX-positive group versus $14 \%$ in the NTX-negative group $(P=$ $0.14)$. When mortality was examined with respect to BMI, no association was found $(P=0.80)$. Examination of mortality with respect to the average $\mathrm{mg} / \mathrm{kg}$ dose, showed no significant differences. Using ABW, patients who died within 30 days received an average dose of $1.79 \mathrm{mg} / \mathrm{kg}$ versus the $2.08 \mathrm{mg} / \mathrm{kg}$ of patients who

TABLE 3 Multivariate analysis for independent predictors for colistinassociated NTX

\begin{tabular}{llll}
\hline Parameter & OR & $95 \%$ CI & $P$ value \\
\hline BMI of $\geq 31.5 \mathrm{~kg} / \mathrm{m}^{2}$ & 3.1 & $1.15-8.35$ & 0.025 \\
Diabetes & 2.11 & $0.84-5.29$ & 0.112 \\
Length of stay (days) prior to CMS & 1.04 & $0.99-1.08$ & 0.078 \\
Age (yr) & 1.08 & $1.00-1.17$ & 0.045 \\
\hline
\end{tabular}


(A)

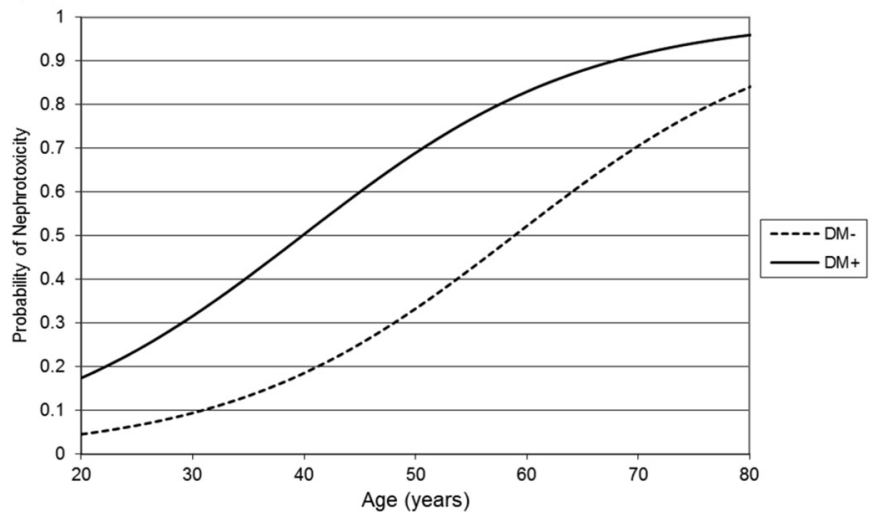

(B)

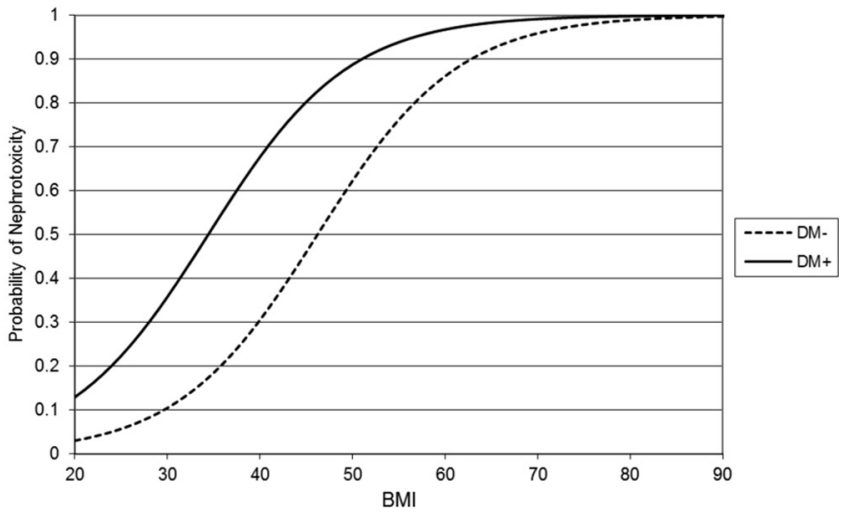

FIG 3 With or without concomitant diabetes mellitus (DM), the probability of NTX versus age in a patient with a BMI of $\geq 31.5 \mathrm{~kg} / \mathrm{m}^{2}$ (A) and the probability of NTX versus BMI in a patient 54 years of age (B).

lived $(P=0.13)$. Using IBW, patients who died within 30 days received an average dose of $3.01 \mathrm{mg} / \mathrm{kg}$ versus the $3.2 \mathrm{mg} / \mathrm{kg}$ of the surviving group $(P=0.5)$.

A respiratory source and a bloodstream source were identified in $50 \%$ and $38 \%$ of the patients, respectively. MDR Acinetobacter baumannii was present in $88 \%$ of the cases, and MDR Pseudomonas aeruginosa was present in $12 \%$. Three patients developed CMS resistance on therapy, and all survived.

\section{DISCUSSION}

This retrospective, nested case-control study shows a high NTX rate of nearly $50 \%$ in overweight and obese adult patients receiving intravenous CMS. The risk of NTX was particularly high in patients with predictors of NTX that include a BMI of $\geq 31.5$ $\mathrm{kg} / \mathrm{m}^{2}$ (independently), older age, diabetes, and a longer duration of hospitalization before CMS receipt. This is the largest study to date of a defined patient population that is representative of an important patient group for which CMS is used worldwide.

The incidence of acute kidney injury, as defined by the RIFLE criteria, revealed a high proportion of patients with failure $(80 \%)$. In the study by DeRyke et al. (4), which included nonoverweight and nonobese patients, 5 of 10 patients who developed NTX were categorized as failure. In analyses performed by Hartzell et al. (12) and Pogue et al. (23), which also included nonoverweight or nonobese patients, failure was found in less than $15 \%$ of the patients experiencing NTX. One contributor to this finding may be the large proportion (95\%) of critically ill patients in our study. However, a study by Doshi et al. (5) investigated solely critically ill patients of all weight categories on CMS and found failure in only $13 \%$ of the patients with acute kidney injury. Another factor to consider based on previous findings by DeRyke et al. is the dosing weight. We found the use of ABW for dosing and excessive dosing due to the use of ABW to be frequent throughout the study at $64 \%$ and $92 \%$, respectively. Such excessive dosing may have contributed to the degree of acute kidney injury among patients in whom NTX occurred.

In-hospital mortality rates have not previously been described in this patient population, and data including nonoverweight or nonobese patients are limited (23). Our finding of a 40\% 30-day all-cause in-hospital mortality rate in the NTX group is similar to the $37 \%$ found in a recent study that included nonoverweight or nonobese persons (23). These high mortality rates likely represent the at-risk populations acquiring MDR Gram-negative infections, for whom addition of NTX to multiple other comorbid conditions may raise an already high baseline mortality rate.

When approaching antibiotic dosing in obese patients, clinicians should be mindful of the complex relationship between antibiotic pharmacokinetics and body size indices $(7,22)$. Obesity can effect drug factors such as distribution, protein binding, metabolism, and clearance. Selecting an appropriate approach to renal function measurement is also challenging (3). The problem may be further aggravated particularly by the volume status of obese critically ill patients (20). As the extravascular space increases, drug distribution is altered. Given the unique history of CMS, this dilemma remains a major concern for clinicians. Colistin is a metabolite of the prodrug CMS, and the pharmacokinetic behavior of metabolites is very difficult to characterize without administering both the parent drug and the preformed metabolite on different occasions. We are unaware of such studies with humans, and these data are urgently needed.

Excessive dosing of CMS (use of ABW for dosing) may result in an increased risk of NTX (4) and more-severe acute kidney injury. Underdosing of CMS (use of IBW) may increase the chance of treatment failure (11) and the development of bacterial resistance (9). In our study, dosing weights were not found to be linearly associated with increased rates of NTX; however, a BMI of $\geq 31.5$ $\mathrm{kg} / \mathrm{m}^{2}$ was. Further, in our study, lower average $\mathrm{mg} / \mathrm{kg}$ doses were given to patients who died. Recently published studies regarding CMS pharmacokinetics suggest that this could be due to colistin levels in vivo not reaching necessary MIC breakpoints (2). The use of loading doses may provide a solution to this problem (11). These points should be considered especially when clinicians treat severely ill patients or those with infections due to organisms with high MICs.

In regard to CMS resistance, patients who developed CMSresistant organisms on therapy $(n=3)$ received excessive doses and did not die. One of these patients experienced NTX with the classification of failure based on RIFLE criteria. Conclusions cannot be drawn from this small sample.

Limitations of this study are that it was retrospective and observational and was completed in a single center. In addition, an institutionally standardized dosing protocol for intravenous CMS 
was not dictated during the study period. Prescriber preference could contribute to potential confounding through the use of higher doses in patients perceived as more severely ill. However, loading doses were not used in any patients, contributing to uniformity. In regard to kidney function, extensive investigation regarding a history of acute kidney injury prior to CMS therapy outside 72 h of therapy initiation was not completed, which could have identified further risk factors or confounding causes of NTX. Finally, using the RIFLE criteria, chronic kidney injury could not be assessed due to the lack of data.

Despite the limitations noted, this study has several strengths. It included a well-characterized study population with clinical and laboratory information available for analysis. Research exclusive to overweight and obese patients is notably limited yet particularly relevant. Definitions (RIFLE, NTX, dosing categorization, etc.) are consistent with recent literature. Additionally, this evaluation included a large percentage of critically ill patients with either $A$. baumannii or $P$. aeruginosa infection and therefore evaluated the effects of CMS on NTX in a specific patient population. Furthermore, many findings, including age as a predictor of CMS-associated NTX, are consistent with previous literature. Finally, we believe this is the first study to evaluate CMS-associated NTX in overweight and obese patients using the RIFLE criteria.

In conclusion, overweight and obese patients receiving intravenous CMS should be monitored closely for NTX. Patients with a BMI of $\geq 31.5 \mathrm{~kg} / \mathrm{m}^{2}$, increased age, diabetes, and longer hospitalization prior to receipt of CMS may be at particular risk. Prospective, randomized, controlled trials are warranted to further examine NTX incidence and predictors and appropriate dosing strategies for this patient population to avoid toxicity and development of bacterial resistance and achieve a clinical cure.

\section{REFERENCES}

1. Bellomo R, et al. 2004. Acute renal failure-definition, outcome measures, animal models, fluid therapy and information technology needs: the Second International Consensus Conference of the Acute Dialysis Quality Initiative (ADQI) Group. Crit. Care 8:R204-R212.

2. Couet W, Grégoire N, Marchand S, Mimoz O. 2012. Colistin pharmacokinetics: the fog is lifting. Clin. Microbiol. Infect. 18:30-39.

3. Demirovic JA, Pai AB, Pai MP. 2009. Estimation of creatinine clearance in morbidly obese patients. Am. J. Health Syst. Pharm. 66:642-648.

4. DeRyke CA, Crawford AJ, Uddin N, Wallace MR. 2010. Colistin dosing and nephrotoxicity in a large community teaching hospital. Antimicrob. Agents Chemother. 54:4503-4505.

5. Doshi NM, Mount KL, Murphy CV. 2011. Nephrotoxicity associated with intravenous colistin in critically ill patients. Pharmacotherapy 31 : 1257-1264.

6. Evans ME, Feola DJ, Rapp RP. 1999. Polymyxin B sulfate and colistin: old antibiotics for emerging multiresistant gram-negative bacteria. Ann. Pharmacother. 33:960-967.
7. Falagas ME, Karageorgopoulos DE. 2010. Adjustment of dosing of antimicrobial agents for bodyweight in adults. Lancet 375:248-251.

8. Falagas ME, Kasiakou SK. 2006. Toxicity of polymyxins: a systematic review of the evidence from old and recent studies. Crit. Care 10:R27.

9. Falagas ME, et al. 2010. Colistin therapy for microbiologically documented multidrug-resistant Gram-negative bacterial infections: a retrospective cohort study of 258 patients. Int. J. Antimicrob. Agents 35:194199.

10. Flegal KM, Carroll MD, Ogden CL, Curtin LR. 2010. Prevalence and trends in obesity among US adults, 1999-2008. JAMA 303:235-241.

11. Garonzik SM, et al. 2011. Population pharmacokinetics of colistin methanesulfonate and formed colistin in critically ill patients from a multicenter study provide dosing suggestions for various categories of patients. Antimicrob. Agents Chemother. 55:3284-3294.

12. Hartzell JD, et al. 2009. Nephrotoxicity associated with intravenous colistin (colistimethate sodium) treatment at a tertiary care medical center. Clin. Infect. Dis. 48:1724-1728.

13. JHP Pharmaceuticals, LLC. 2011. Coly-Mycin M parenteral (colistimethate for injection, USP) package insert. JHP Pharmaceuticals, LLC, Parsippany, NJ.

14. Kellum JA, Bellomo R, Ronco C. 2008. Definition and classification of acute kidney injury. Nephron Clin. Pract. 109:c182-c187.

15. Kim J, Lee K-H, Yoo S, Pai H. 2009. Clinical characteristics and risk factors of colistin-induced nephrotoxicity. Int. J. Antimicrob. Agents 34: $434-438$.

16. Koch-Weser J, et al. 1970. Adverse effects of sodium colistimethate. Ann. Intern. Med. 72:857-868.

17. Kwon J-A, et al. 2010. Predictors of acute kidney injury associated with intravenous colistin treatment. Int. J. Antimicrob. Agents 35:473-477.

18. Li J, et al. 2006. Colistin: the re-emerging antibiotic for multidrugresistant Gram-negative bacterial infections. Lancet Infect. Dis. 6:589601.

19. Lim LM, et al. 2010. Resurgence of colistin: a review of resistance, toxicity, pharmacodynamics, and dosing. Pharmacotherapy 30:1279-1291.

20. McKenzie C. 2011. Antibiotic dosing in critical illness. J. Antimicrob. Chemother. 66(Suppl 2):ii25-ii31.

21. Nation RL, Li J. 2009. Colistin in the 21st century. Curr. Opin. Infect. Dis. 22:535-543.

22. Pai MP, Bearden DT. 2007. Antimicrobial dosing considerations in obese adult patients. Pharmacotherapy 27:1081-1091.

23. Pogue JM, et al. 2011. Incidence of and risk factors for colistin-associated nephrotoxicity in a large academic health system. Clin. Infect. Dis. 53: $879-884$.

24. Pogue JM, Marchaim D, Kaye D, Kaye KS. 2011. Revisiting “older" antimicrobials in the era of multidrug resistance. Pharmacotherapy 31: 912-921.

25. Salazar DE, Corcoran GB. 1988. Predicting creatinine clearance and renal drug clearance in obese patients from estimated fat-free body mass. Am. J. Med. 84:1053-1060.

26. Spapen H, Jacobs R, Van Gorp V, Troubleyn J, Honoré PM. 2011. Renal and neurological side effects of colistin in critically ill patients. Ann. Intensive Care 1:14.

27. WHO 2004, posting date. World Health Organization body mass index classification. World Health Organization, Geneva, Switzerland. http: //apps.who.int/bmi/index.jsp?introPage=intro_3.html.

28. X-Gen Pharmaceuticals, Inc. 2010. Colistimethate (colistin) package insert. X-Gen Pharmaceuticals, Inc., Big Flats, NY. 